\title{
RIFORMA DEL METODO E FINI MORALI IN CARTESIO
}

Gregorio Piaia*

SÍNTESE - Facilmente lê-se Descartes como autor de um projeto de reforma do conhecimento, estranhando-se mesmo a presença da moral, na parte terceira do Discurso. Uma análise aprofundada do projeto cartesiano mostra, entretanto, que na economia complessiva intelectual projetada por ele, a moral representa ao mesmo tempo o pressuposto e o ponto alto de tal reforma.

"Come sappiamo, Signori, il giorno, il mese, l'anno in cui la filosofia greca è stata messa al mondo [il riferimento è alla nascita di Socrate], così sappiamo, con la stessa certezza e con maggiori dettagli ancora, il giorno e l'anno in cui la filosofia moderna è nata. [...] È dunque dal 1637 che data la filosofia moderna! E qual è, Signori, il titolo di quest'opera eminentemente storica? Il metodo. Vi ho detto che Socrate non aveva affatto avuto un sistema; vi dirò che importa assai poco che Cartesio ne abbia avuto uno. Il pensiero di Cartesio che appartiene alla storia è quello del suo metodo. Socrate era la riflessione libera; Cartesio è la riflessione libera innalzata all'altezza di un metodo, anzi il metodo nella sua forma più severa". ${ }^{1}$

Ė con questo tono enfatico che Victor Cousin, nella seconda lezione del suo corso di storia della filosofia alla Sorbona (24 aprile 1828), consacrava un ritratto di Cartesio ch'era già stato abbozzato nella cultura settecentesca e che si sarebbe fissato in profondità nell'immaginario filosofico dell'Otto-Novecento, pur nella varietà delle interpretazioni. Domina in questo ritratto, accanto al topos che vede in Cartesio l'"iniziatore" della filosofia moderna, il tema della centralità del "metodo", colto ed esaltato nella sua struttura formale, anzi nel suo valore simbolico e nella sua portata futura, più che nei contenuti che ne costituirono lo sbocco 'materiale'. La qual cosa - vale la pena ricordarlo - andava contro gl'intendimenti dello stesso Cartesio, per il quale il breve Discours de la méthode pour bien conduire sa raison et chercher la vérité dans les sciences doveva servire da introduzione alle successive, ampie (e per lui ben più importanti) trattazioni sulla diottrica,

* Università di Padova.

1 V. COUSIN, Cours de philosophie. Introduction à l'historie de la philosophie, texte revu par P. Vermeren, Paris 1991, pp. 59-60.

\begin{tabular}{|l|l|l|l|l|l|}
\hline VERITAS & Porto Alegre & v. 43 & $n^{2} 2$ & Junho 1998 & p. 329-339 \\
\hline
\end{tabular}


sulle meteore e sulla geometria analitica, che nell'edizione di Leida, apparsa anonima l'8 giugno 1637, occupano oltre 500 pagine in $-4^{\circ}$.

In realtà il Discours ci appare una sorta di vetrina in cui l'autore espone, in una cornice autobiografica, un variegato campionario dei filosofici prodotti legati all'applicazione del suo metodo, dal progetto di una radicale riforma del sapere alle prove della dimostrazione di Dio, dalla tesi sulla circolazione del sangue alla teoria dell'automatismo animale. Un campionario che non mira soltanto a 'vendere', ma anche a saggiare (nella "Parte quinta") le reazioni delle autorità politicoreligiose in vista della stampa dell'impegnativa opera Le monde ou Traité de la lumière, sulla quale poi - com'è noto - il prudente Cartesio soprassedette sino alla fine dei suoi giorni, memore della sorte toccata a Galileo.

Fra i 'prodotti' esposti dal filosofo v'è pure, nella "Parte terza", la morale, che a prima vista risulta però ai margini o addirittura fuori campo, anche perché l'attributo "provvisoria" (une morale par provision) sembra stonare con la mentalità geometrica dell'autore e con il carattere rigoroso e definitivo delle regole metodiche. Questa terza parte del Discours parrebbe quasi una toppa messa li per prevenire possibili accuse di sovvertimento politico o religioso, garantendo cosi quella tranquillità di vita cui l'autore teneva altamente. "Benchè si riconosca l'estrema importanza del contributo cartesiano alla filosofia della conoscenza - nota in proposito John Cottingham -, il suo contributo alla filosofia pratica è generalmente giudicato trascurabile": 2 trascurabile rispetto alla communis opinio e alla mole enorme della bibliografia cartesiana, giacché, per quanto concerne la morale, non mancano certo i volumi e i saggi dedicati a tale problematica. ${ }^{3}$

In effetti nell'economia complessiva della riforma intellettuale progettata da Cartesio - e imperniata su una rigorosa deduzione dei vari rami del sapere dai primi principi - la morale rappresenta ad un tempo il presupposto e il culmine. E quanto appare con chiarezza nella "Préface" alla traduzione francese dei Principia philosophiae (1647), ov'è indicato l'"ordine" che l'uomo-tipo deve seguire per un itinerario che non è soltanto conoscitivo, ma mira a perfezionare l'intera natura umana: egli deve "innanzi tutto cercare di formarsi una morale, che possa bastare per regolare le azioni della sua vita, poiché ciò non ammette nessuna proroga, e noi dobbiamo sopra tutto cercare di viver bene". ${ }^{4} \mathrm{E}$ la medesima istanza sottesa alla morale provvisoria del Discours, che, lungi dall'esaurirsi in un espediente tattico, si rivela la pre-condizione stessa di una corretta riforma intellettuale: non solo in base all'adagio primum vivere, ma anche perché un'esistenza in balia dell'arbitrio e del disordine morale pregiudica ogni indagine razionale; il binomio romantico genio-sregolatezza è inconcepibile per Cartesio, che non a caso vive nell'âge classique...

La morale ricompare al termine del percorso autoformativo, illustrato con una rinnovata versione della metafora dell'arbor scientiarum. "Cosi tutta la filosofia è

2 J. COTTINGHAM, Cartesio, tr. it., Bologna 1991, p. 196.

3 Cfr. (oltre al prezioso lavoro di G. SEBBA, Bibliographia Cartesiana. A critical guide to the Descartes, 1800-1960, The Hague 1964) G. CRAPULLI, Introduzione a Descartes, Roma-Bari 1992, pp. 278-280. Fra gli studi più recenti segnaliamo l'articolo dello stesso J. COTTINGHAM, Cartesian Ethics: Reason and the Passions, "Revue internationale de philosophie", 50 (1996), pp. 193-216.

CARTESIO, Opere, a cura di E. Garin, Bari 1967, II, p. 19 (ed. Adam-Tannery, IX-2, p.3). 
come un albero, di cui le radici sono la metafisica, il tronco è la fisica, e i rami che sortono da questo tronco sono tutte le altre scienze, che si riducono a tre principali, cioè la medicina, la meccanica e la morale, intendo la più alta e perfetta morale, che, presupponendo un'intera conoscenza delle altre scienze, è l'ultimo grado della saggezza (le dernier degré de la sagesse)". ${ }^{5}$ Sono queste discipline che rappresentano "la principale utilità della filosofia", come Cartesio aveva già precisato nella definizione introduttiva della filosofia come "studio della saggezza". ${ }^{6}$ Una definizione onnicomprensiva, che si estende a tutto ciò che (essendo specifico dell'uomo come res cogitans, ossia pensiero e azione cosciente e responsabile) ci distingue dai "bruti".?

Se per Aristotele la massima realizzazione dell'uomo consisteva nell'esercizio delle virtù dianoetiche e quindi nella contemplazione delle cause prime e dei principi ultimi, per Cartesio la ricerca delle "prime cause, cioè dei principi" non è fine a se stessa, ma è in funzione dei frutti ultimi dedotti da tali principi e fra i quali primeggiano i frutti morali: eredità della filosofia ellenistica, in cui lo studio della logica e della fisica era finalizzato all'etica; un'eredità mediata dalla cultura dell'umanesimo, e poi arricchita e trasfigurata dalle applicazioni che l'incipiente rivoluzione scientifica già lasciava intravvedere, e che Bacone aveva profeticamente illustrato nella Nuova Atlantide. Non a caso, richiamandosi esplicitamente al suo Discours e alle tre opere che l'accompagnavano, Cartesio scende nel dettaglio dicendo ch'era suo intento "far vedere che si poteva progredire assai nella filosofia, per giungere per suo mezzo alla conoscenza delle arti che sono utili alla vita, perché l'invenzione delle lenti d'ingrandimento [...] è una delle più difficili che siano state mai oggetto di ricerca". ${ }^{8}$

Stupisce, a dire il vero, che nello schema del sapere qui tracciato manchi quel prodotto tipicamente umano che sono le leggi ordinatrici della vita associata, tanto più che in quella stessa pagina Cartesio pone un rapporto diretto tra sviluppo della civiltà e sviluppo della filosofia, e si spinge ad esaltare il beneficio che i "veri filosofi" arrecano allo Stato. ${ }^{9}$ Avremo modo di ritornare più avanti sui rapporti tra la filosofia e l'autorità costituita, depositaria delle leggi civili e religiose; c'interessa ora rilevare alcune significative assonanze con la quattrocentesca "disputa sulle

5 CARTESIO, Opere, II, p. 19 (ed. Adam-Tannery, IX-2, p. 14).

6 " [...] ce mot Philosophie signifie l'estude de la Sagesse, et [...] par la Sagesse on n'entend pas seulement la prudence dans les affaires, mais une parfaite connoissance de toutes les choses que l'homme peut sçavoir, tant pour la conduite de sa vie, que pour la conservation de sa santé et l'invention de tous les arts; et [...] afin que cette connoissance soit telle, il est necessaire qu'elle soit déduite des premieres causes [...]" (ed. Adam-Tannery, IX-2, p. 2).

7 "C'est proprement avoir les yeux fermez, sans tascher jamais de les ouvrir, que de vivre sans philosopher; et le plaisir de voir toutes les choses que notre veuë découvre n'est point comparable à la satisfaction que donne la connoissance de celles qu'on trouve par la Philosophie; et enfin cet estude est plus necessaire pour regler nos moeurs, et nous conduire en cette vie, que n'est l'usage de nos yeux pour'guider nos pas" (ed. Adam-Tannery, IX-2, pp. 3-4). CARTESIO, Opere, II, p. 20 (ed. Adam-Tannery, IX-2, p. 15).

9 "Y'aurois en suite fait considerer l'utilité de cette Philosophie, et monstré que, puis qu'elle s'estend à tout ce que l'esprit humain peut sçavoir, on doit croire que c'est elle seule qui nous distingue des plus sauvages et barbares, et que chaque nation est d'autant plus civilisée et polie que les hommes y philosophent mieux; et ainsi que c'est le plus grand bien qui puisse estre en un Estat, que d'avoir de vrais Philosophes" (ed. Adam-Tannery, IX-2, p. 3). 
arti", che verteva sulla superiorità del diritto oppure della medicina. Al contrario di Coluccio Salutati (che rivendicava il primato della giurisprudenza, fondata su princìpi non empirici ma interni all'uomo e quindi universali) Poggio Bracciolini, ad es., sottolineava l'arbitrarietà delle leggi civili, buone solo per il popolo minuto, giacché "gli uomini gravi, saggi, modesti, non hanno bisogno di leggi. Si sono dati da sé la legge del vivere onesto, educati alla virtù e al buon costume dalla natura e dallo studio": un ritratto che si attaglierebbe al nostro Cartesio, per il quale l'obbedienza alle leggi e ai costumi del proprio paese risponde a un'esigenza generale di ordine e di sicurezza, garanzia esteriore per un perfezionamento individuale in cui il saggio diventa legge a se stesso. Dal canto suo il celebre averroista padovano Nicoletto Vernia aveva proclamato il primato della scientia naturalis sulla politica, escludendo recisamente che quest'ultima possa condurre alla "vera felicità dell'uomo", posta nella cognitio Dei et aliarum intelligentiarum, cui si può pervenire solo attraverso la scienza naturale. E Antonio de Ferrariis (detto il Galateo) affermò con convinzione il primato della medicina, intesa come scienza complessiva della natura (in quanto studia gli elementi, i luoghi e i climi, le virtú delle erbe e dei metalli...) e di quella mirabile costruzione che è il corpo umano (opificium humani corporis): essa è una scienza contemplativa e non solo pratica, mentre la giurisprudenza e la politica si risolvono tutte nell'azione, e la contemplazione $\grave{e}$, aristotelicamente, superiore all'azione $[\ldots]^{10}$

Certo, Cartesio rifiuta l'impostazione aristotelica, ma a ben vedere il suo ideale di sagesse, in cui la soddisfazione che nasce da una conoscenza bene fondata si coniuga con le applicazioni utili all'anima (la morale), al corpo (la medicina) e alla vita associata (le arti meccaniche), senza toccare l'ambito strettamente giuridico, non è lontano dagli autori sopra citati: è un'eredità rinascimentale (meglio, di un certo Rinascimento) che possiamo cogliere in lui, senza per questo dover riproporre la tesi - fortunata ma anche contestata - di chi poneva un legame di derivazione fra l'aristotelismo eterodosso italiano e il libertinismo francese. ${ }^{11}$

Il richiamo alla lettera-prefazione dei Principes ci consente di rileggere il Discours de la méthode in maniera alquanto diversa da quella in uso nelle nostre scuole, che risente fortemente dell'interpretazione hegeliana, tutta incentrata sull'unità tra pensiero ed essere e sulla fondazione logico-metafisica dell'interiorità, quale premessa storica e speculativa all'idealismo tedesco. È una lettura solitamente basata sulle regole del metodo, sul cogito e sulle prove dell'esistenza di Dio, con qualche accenno alla critica della cultura tradizionale (quale introduzione al metodo) e alla morale provvisoria, intesa per lo più come un atteggiamento di comodo. Le parti V e VI sono regolarmente omesse, perché - si sa - il tempo man$\mathrm{ca}$, e poi esse sembrano discostarsi dal 'razionalismo cartesiano' così ben codificato nei manuali e manualetti, e registrato a suo tempo dallo scrittore Gustave

10 Sugli autori qui citati si veda E. GARIN, Storia della_filosofia italiana, III ed., Torino 1978, I, pp. 272 279 e la relativa bibliografia.

11 Cfr. in particolare J.R. CHARBONNEL, La pensée italienne au XVIe siècle et le courant libertin, Paris 1919. 
Flaubert nel suo ironico dizionarietto di luoghi comuni, dove alla voce Descartes compare l'immancabile "cogito ergo sum."...12

La chiave di lettura alternativa che qui proponiamo è costituita, per l'appunto, dal nesso organico fra la ricerca di un nuovo metodo e la prospettiva morale, sulla base di quel principio intellettualistico che viene formulato esplicitamente e con forte convinzione nel corso della "Parte terza" del Discours: "La nostra volontà, infatti, per sé, non è portata a seguire o a fuggire cosa alcuna se non in quanto l'intelletto (notre entendement) gliela presenta buona o cattiva; e dunque basta giudicar bene per bene fare (il suffit de bien juger, pour bien faire), e giudicare il meglio che si può per fare anche in tutto il proprio meglio, ossia per acquistare tutte le virtù e insieme tutti gli altri possibili beni. E non si può non essere contenti quando si è certi che è cosi". ${ }^{13}$ Questa prospettiva - sia pure in filigrana - è presente nel Discours de la méthode assai prima della "Parte terza", espressamente dedicata alla questione etica. Il nesso metodo-morale, che già ispirava il titolo primitivo del Discours (Projet d'une science universelle qui puisse élever notre nature à son plus haut degré de perfection) si coglie infatti nelle considerazioni iniziali su le bon sens ou la raison, una facoltà distribuita in tutti gli uomini, ma impiegata in maniere diverse, il che dà luogo alla diversità di opinioni. "Poiché nota Cartesio - non basta avere un buon ingegno (l'esprit bon): quel che più importa è di applicarlo bene (de l'appliquer bien)". È' una pregiudiziale metodica che viene subito proiettata, senza mediazioni, al centro della sfera morale, dominato dall'opposizione virtù-vizio: "Le anime più grandi sono capaci dei più grandi vizi come delle più grandi virtù; e quelli che seguono sempre la via dritta, anche se camminano assai lentamente, possono andare molto più innanzi di coloro che più corrono e più se ne allontanano". ${ }^{14}$

Il concetto squisitamente morale di "vizio" è dunque correlato agli "errori" nel metodo conoscitivo, e tali errori - dirà Cartesio nella parte seconda - sono favoriti dal numero eccessivo delle regole, come avveniva nella logica peripateticoscolastica. E qui ritorna l'accenno al "vizio", in una prospettiva non soltanto individuale ma riferita alla società organizzata, ossia allo "Stato", il cui corpus legislativo è significativamente paragonato alle regole metodiche (un'eco degli studi in utroque iure compiuti a Poitiers?): come nello Stato meno leggi ci sono, meno si

12. Le dictionnaire des idées reçues, ou le catalogue des opinions chic, in G. FLAUBERT, Oeuvres, Paris 1959, II, p. 1004 (si veda pure, a p. 1015, la voce Jansénisme: “On ne sait pas ce que c'est, mais il est très chic d' en parler"...).

13 Discorso sul metodo, p. 75 (ed. Gilson, p. 28). L'intellettualismo etico verrà ribadito con vigore nella lettera a Elisabetta del Palatinato (4 agosto 1645) e poi nel trattato Les passions de l'âme, ad esempio negli articoli XLVIII-XIIX, CXLIV e CXLVIII. La conclusione di quest'ultimo articolo (il quale chiude a sua volta la seconda parte del trattato) offre un quadro sintetico in cui ritroviamo i concetti (e i termini) tipici della morale cartesiana, compresa la definizione di 'virtù': "Car quiconque a vescu en telle sorte, que sa conscience ne luy peut reprocher qu'il ait jamais manqué à faire toutes les choses qu'il a jugées estre les meilleures (qui est ce que je nomme icy suivre la vertu), il en reçoit une satisfaction, qui est si puissante pour le rendre heureux, que les plus violens effors des passions n'ont jamais assez de pouvoir pour troubler la tranquillité de son âme" (ed. Adam-Tannery, XI, p. 442).

14 DESCARTES, Discorso sul metodo, a cura di A. Carlini, XXVIII ed., Roma-Bari 1991, p. 41 (Discours de la méthode, texte et commentaire par E. Gilson, Paris 1962, p. 2). 
favoriscono i vizi, cosi, minori regole ci sono nel metodo, minori sono le possibilità di enrore. ${ }^{15}$

Lo stretto legame con la filosofia pratica riaffiora quando Cartesio ricorda di aver sempre avuto "un ardente desiderio d'imparare a distinguere il vero dal falso per veder chiaro nelle mie azioni e camminare con sicurezza nella vita"; un'esigenza cui fa subito da pendant negativo la constatazione della grande disparità di comportamenti e costumi (moeurs) fra gli uomini, analoga alla disparità di opinioni tra i filosofi. ${ }^{16}$ Queste enunciazioni di carattere più generale si alternano a minuti riferimenti agli stati d'animo, in cui la dimensione psicologica assume i connotati di una vera e propria "etica della conoscenza": l'extrême satisfaction per i progressi nella ricerca della verità, accompagnata però dalla défiance - più che dalla présomption - nel giudicare se stesso (una défiance che si estende anche ai giudizi degli amici, a volte troppo benevoli nei nostri confronti); la presunzione di chi s'impiccia a dar precetti agli altri, rischiando poi il biasimo; il rifiuto di acquistare gloria in campo scientifico grazie a "falsi titoli" (pur non condividendo il disprezzo dei Cinici per la gloria umana); la denuncia delle mauvaises doctrines di maghi, astrologi ed alchimisti (frutto di impostures) e della vanterie di quanti fanno professione di sapere quel che non sanno; la critica dell'atteggiamento mentale di chi, chiuso nel suo studiolo, si dedica a "speculazioni" prive di effetti e che lo rendono tanto più vanitoso quanto più si allontana dal senso comune... ${ }^{17}$

La corrispondenza fra il piano conoscitivo e quello etico non è però totale e assoluta. Se è metodologicamente corretto sospendere il giudizio in attesa di pervenire alla verità, per Cartesio l'irresolutezza nel comportamento non è ammissibile, perché compromette la felicità del nostro vivere (vivre [...] le plus heureusement que je pourrais), ed è su questo divario che s'innesta il discorso sulla morale provvisoria, quale prima applicazione del nuovo metodo ${ }^{18}$ Come già rilevò efficacemente il Gilson, questa morale è tale non perché si contrappone a quella "definitiva": anzi, essa "può essere definitiva" - e in questi termini è ripresentata nella lettera a Elisabetta del Palatinato del 4 agosto 1645 -, "ma noi non sappiamo ancora se lo sia", perché la più elevata conoscenza dei principi morali è possibile solo dopo aver esaurito le altre scienze, e poi perché "l'ordine del vero" non coincide sempre con "l'ordine del bene". ${ }^{19}$

In tale modo la nozione di 'verosimile' o di 'probabile', espunta dal processo conoscitivo, è ammessa nella sfera del comportamento, ove può anzi raggiungere lo stesso grado di certezza della verità, assurgendo a seconda massima della morale provvisoria: " $\mathrm{E}$ cosi, quando, come spesso accade nella vita, le azioni non ammettono indugio, e non sia in poter nostro discernere le opinioni più vere, non

15 "Et comme la multitude des lois fournit souvent des excuses aux vices, en sorte qu'un État est bien mieux réglé lorsque, $n$ 'en ayant que fort peu, elles y sont fort étroitement observées; ainsi, au lieu de ce grand nombre de préceptes dont la logique est composée, je crus que j'aurais assez des quatre suivants, pourvu que je prisse une ferme et constante résolution de ne manquer pas une seule fois à les observer" (ed. Gilson, p. 18). Ma la costanza è anche una virtù morale e non solo un requisito metodologico...

16 Discorso sul metodo, pp. 51-52 (ed. Gilson, p. 10).

17 Discorso sul metodo, pp. 43, 44, 50, 51 (ed. Gilson, pp. 3, 4, 9, 10).

18 Discorso, p.68 (ed. Gilson, p. 22).

19 Discours, ed. Gilson, "Commentaire historique", pp. 230-234. 
c'è dubbio alcuno che dobbiamo seguire le più probabili; e se pure non notiamo maggiori probabilità nelle une che nelle altre, bisogna bene nondimeno determinarci per alcune, e considerarle perciò in sèguito, praticamente (en tant qu'elles se rapportent à la pratique), non più come dubbie, anzi come verissime e certissime (très vraies et très certaines), in quanto tale è la ragione che ce le ha fatte preferire alle altre". ${ }^{20}$ Come dire che l'esigenza della ragion pratica di mantenere la risolutezza e la costanza nel nostro agire (per non cadere in preda ai "pentimenti e rimorsi [les repentirs et les remords] che sogliono agitare la coscienza degli spiriti deboli e sempre oscillanti") prevale sul criterio dell'evidenza conoscitiva: la scienza è in funzione della felicità umana, ma la complessità della vita e le esigenze immediate possono far sì che l'equilibrio della nostra condotta - che è condizione di una vita felice - faccia talora aggio sulla rigorosa ricerca della verità.

Primum vivere, dunque...; sed bene vivere, ossia vivere beate, per usare l'espressione di Seneca, che Cartesio nella ricordata lettera a Elisabetta traduce "vivere felicemente (vivre heureusement)", distinguendo poi subito tra la "felicità (heur)" e la "beatitudine (béatitude)": "la felicità dipende esclusivamente dalle cose che sono fuori di noi, onde deriva che sono stimati piuttosto felici che saggi coloro a cui è capitato qualche bene che non si sono procurati da sé. La beatitudine, invece, consiste, mi sembra, in una perfetta contentezza dello spirito (en un parfait contentement d'esprit) e in una soddisfazione interiore che ordinariamente non posseggono coloro che più sono favoriti dalla fortuna, mentre i saggi l'acquistano senza la fortuna. Così vivere beate, ossia vivere in beatitudine, non è altro che avere lo spirito perfettamente contento e soddisfatto". ${ }^{21} \mathrm{Ne}$ consegue che la "sovrana contentezza (souverain contentement)" in cui consiste la piena felicità non deriva dai beni che non dipendono da noi (onori, ricchezze, salute) bensi dalla "virtù" e dalla "saggezza", che sole sono in nostro potere. Sicché Cartesio giunge alla paradossale - per noi - conclusione che, "prendendo la gioia di ciascuno come la pienezza e il compimento dei suoi desideri regolati secondo ragione, [...] i più poveri, i più perseguitati dalla fortuna o maltrattati dalla natura possono essere completamente felici", nel senso, ovviamente, della béatitude. È questo principio che sottende in particolare la terza massima della morale provvisoria, quale viene riproposta alla principessa Elisabetta; ed è un principio che nel Discours era stato enunciato con grande chiarezza, in conformità con l'intellettualismo etico sopra evidenziato. ${ }^{22}$

La chiave della felicità vera (la béatitude) sta dunque nella piena padronanza di sé, ovvero nell'esercizio delle virtù, come verrà ribadito nel trattato Les passions de l'âme (1649), in cui Cartesio tenta di stabilire un collegamento fra il controllo razionale degli stati emotivi, debitamente analizzati e classificati, e i meccanismi

20 Discorso, pp. $71-72$ (ed. Gilson, p. 25).

21 CARTESIO, Opere, II, p. 546 (ed. Adam-Tannery, IV, p. 264).

22 "Car notre volonté ne se portant naturellement à désirer que les choses que notre entendement lui représente en quelque façon comme possibles, il est certain que, si nous considérons tous les biens qui sont hors de nous comme également éloignés de notre pouvoir, nous n'aurons pas plus de regrets de manquer de ceux qui semblent être dus à notre naissance, lorsque nous en serons privés sans notre faute, que nous avons de ne posséder pas les royaumes de la Chine ou du Mexique" (Discours, ed. Gilson, pp. 25-26). 
psico-fisiologici del corpo umano; ed a questo trattato egli aveva alluso nella lettera all'amico Chanut del 15 giugno 1646, in cui dichiara che, "invece di scoprire [con lo studio della medicina] i mezzi per conservare la vita, ho trovato [mediante la riflessione morale, fondata sulla conoscenza della fisica] qualcosa di molto più confortevole e sicuro, cioè il mezzo di non temere la morte; senza per questo essere malinconico [chagrin, oggi diremmo 'depresso'], come lo sono per lo più coloro la cui saggezza è ricavata completamente dagl'insegnamenti altrui e fondata su basi che dipendono solo dalla prudenza e dall'autorità degli uomini”, ossia da fattori estrinseci. ${ }^{23}$

L'influsso della morale stoica è evidente. D'altronde - e qui torniamo alla "Parte terza" del Discours - Cartesio esprime apertamente la sua ammirazione per "quei filosofi che [grazie a un longue exercice e ad una méditation souvent réitéréel hanno potuto in altri tempi sottrarsi all'impero della fortuna e, malgrado i dolori e la povertà, gareggiare in felicità con i loro dèi (disputer de la félicité avec leurs dieux)" ${ }^{24} \mathrm{E}$ non a caso, intrattenendo la principessa Elisabetta sui mezzi con cui acquistare la felicità interiore, proporà la lettura di qualche libro degli antichi, da arricchire con le proprie riflessioni, e fra questi segnalerà il De vita beata di Seneca. ${ }^{25}$ Se poi riandiamo al celebre inizio della "Parte seconda", là dove il filosofo racconta che in un quartiere invernale di Germania, non essendo turbato da cure o da passioni (n'ayant d'ailleurs, par bonheur, aucuns soins ni passions qui me troublassent), poté dedicarsi interamente alla riflessione filosofica, sembra quasi di aver a che fare con una moderna versione del saggio stoico. ${ }^{26}$

Eppure proprio Cartesio, rievocando - poche pagine addietro - gli studi compiuti a La Flèche, aveva demolito i "trattati di morale degli antichi pagani", paragonandoli a "superbi e magnifici palazzi, ma costruiti su sabbia e fango". Anche in questo caso l'allusione è generica, ma è chiaramente riferita agli stoici, ai quali si rimprovera d'elevare al massimo grado le virtù come se fossero l'oggetto più pregevole al mondo, senza però insegnare a sufficienza a conoscerle; anzi, "spesso quello a cui danno un cosi bel nome non è che insensibilità, o orgoglio, o disperazione, o parricidio [con riferimento, forse, all'uccisione di Cesare]". ${ }^{27}$ Analogamente, l'invito ad Elisabetta a leggere Seneca non è esente da critiche al filosofo latino: nella lettera successiva egli giudica poco exacte la trattazione svolta nel $\mathrm{De}$ vita beata, e nella chiusa si richiama nuovamente al nesso "verità-virtù", ossia "intelletto-volontà", che è al centro della sua prospettiva filosofica "[...] mi sembra che Seneca avrebbe dovuto insegnarci tutte le principali verità la cui conoscenza è richiesta per facilitare l'uso della virtù, per regolare i nostri desideri e le nostre passioni e godere così della beatitudine naturale. Questo avrebbe reso il suo libro il migliore e il più utile che un filosofo pagano potesse scrivere"). ${ }^{28}$ Ma ciò presuppone una riforma del sapere (grazie all'elaborazione e all'applicazione di un nuovo metodo, diverso anche dalla logica degli stoici, probabilmente confusa con quella

23 CARTESIO, Opere, II, p. 587 (ed. Adam-Tannery, IV, p. 442).

24 Discorso, pp. $73-74$ (ed. Gilson, p. 26).

25 CARTESIO, Opere, I, p. 545 (ed. Adam-Tannery, IV, pp. 252-253).

26 Discorso, p. 53 (ed. Gilson, p. 11).

27 Discorso, p. 48 (ed. Gilson, pp. 7-8).

28

CARTESIO, Opere, II, pp. 546 e 548. 
peripatetico-scolastica), che è la garanzia per un effettivo perfezionamento, intellettuale e morale ad un tempo.

Il rapporto di Cartesio con lo stoicismo è dunque ambiguo e ambivalente, sospeso com'è fra l'ammirata condivisione di alcuni temi di fondo e la critica a certo astratto estremismo, cui si aggiunge la rivendicazione di una novità ed originalità che è epistemologica più che morale (o, meglio, che è compiutamente morale in quanto rigorosamente 'scientifica'...). Ed è un rapporto reso più complesso dallo sviluppo, fra Cinque e Seicento, di un vero e proprio neostoicismo, che ebbe in Giusto Lipsio il suo alfiere e in Seneca il suo nume tutelare, e che esercito un largo influsso sugli uomini di cultura. ${ }^{29}$ Ad ogni modo, pur in forma autonoma ed 'anomala' rispetto ai neostoici, il debito di Cartesio verso il Portico di Atene, verso Seneca e verso Epitteto rimane profondo. Lo stesso carattere fortemente individuale dell'autoperfezionamento, così com'è teorizzato e vagheggiato nel Discours, sembra rifarsi al modello dell'antico saggio stoico, isolato nella sua libertà interiore e nel suo cosmopolitismo che lo tiene lontano da ogni impegno nel mondo ("più spettatore che attore nelle commedie che vi si rappresentano"), ${ }^{30}$ anche se l'universalità del logos (e del nomos) è principio di un'unità superiore.

In tale prospettiva le precisazioni di Cartesio sul carattere strettamente personale della sua proposta di un nuovo metodo assumono un significato che va oltre l'ovvia precauzione nei confronti del potere politico (e religioso). Ė l'eco dei saggi stoici (o più in generale dell'individualismo ellenistico) che si coglie nella contrapposizione fra le difficoltà non insormontabili che s'incontrano nella riforma della propria condotta intellettuale (e quindi morale) e gli enormi ostacoli che intralciano la "sia pur minima riforma della cosa pubblica (en la réformation des moindres choses qui touchent le public)"; giacché - e l'osservazione è oggi quanto mai attuale - "questi grandi corpi sono difficili a rialzare quando sono abbattuti, o anche solo a tener in piedi quando sono scossi, e le loro cadute sono sempre molto gravi", anche se l'usage finisce per attenuare e correggere le imperfections dello Stato, che risultano alla fine più sopportabili di un radicale cambiamento... Cartesio prende quindi le distanze da "quei caratteri turbolenti ed irrequieti che, non chiamati al maneggio degli affari pubblici né per la loro nascita né per altra fortuna, han sempre in mente nuovi progetti di riforme", ribadendo con umiltà invero eccessiva che "il mio disegno è stato sempre di riformare soltanto i miei pensieri e di edificare in un fondo tutto mio: nulla di piư". ${ }^{31}$

29 Sul neostoícismo tardo rinascimentale e sui rapporti fra la morale di Cartesio e quella stoica cfr. L. ZANTA, La renaissance du Stoïcisme au XVlème siècle, Paris 1914; J. SEGOND, La sagesse cartésienne et la doctrine de la science, Paris 1932, pp. 63-71; J.L. SAUNDERS, Justus Lipsius. The philosophy of Renaissance Stoicismus, New York 1955; A. BRDOUX, Le stoïcisme et son influence, Paris 1966; V. BROCHARD, Descartes stoïcien, in ID., Études de philosophie ancienne et de philosophie modeme, Paris 1966, pp. 320-326; J. DELHEZ, Descartes lecteur de Sénèque, "Latomus", 114 (1970), pp. 392-401; J.-E. d'ANGERS, Recherches sur le stoicisme au XVIe et XVIIe siècles, Hildesheim 1976 (in particolare pp. 453-480: "Sénèque, Epictète et le Stoïcisme dans d'oeuvre de René Descartes"); Atoms, Pneuma and Tranquillity. Epicurean and Stoic themes in European thought, ed. by M.J. Osler, Cambridge (N.Y) 1991.

30 Discorso, p. 76 (ed. Gilson, p. 28).

31 Discorso, pp. 56-57 (ed. Gilson, pp. 14-15). 
L'ossequio del filosofo verso l'autorità costituita, la quale gli consente di "godere di una vita serena e tranquilla (tranquillo otio)", era stato affermato a chiare lettere da Seneca, in opposizione a "chi pensa che i veri filosofi siano arroganti e ribelli (contumaces ac refractarios)". ${ }^{32}$ Ma lo stesso Seneca nella lunga Ep. 90, in contrasto con Posidonío di Apamea, esponente del cosiddetto mediostoicismo, aveva distinto nettamente la filosofia (che siede più in alto ed è "maestra non delle mani, ma delle anime") dalle arti manuali e meccaniche, che con le loro invenzioni danno luogo ad un progresso sociale che è del tutto superfluo, poiché "a soddisfare le esigenze della natura basta la natura stessa (sufficit ad id natura quod poscit)" ${ }^{33}$

Non a Seneca, quindi, ma semmai a Posidonio si può accostare l'interesse di Cartesio per quelle 'applicazioni' che costituiscono il culmine della filosofia stessa, come s'è rilevato nella préface ai Principes. Si tratta di un tema ben presente nel Discours, anche se ai nostri sprovveduti studenti può apparire più baconiano che 'cartesiano'. Già nelle pagine introduttive, dopo aver ricordato fra le sue giovanili acquisizioni culturali il fatto che "le matematiche hanno invenzioni sottilissime, utili assai tanto a contentare i curiosi, quanto a facilitare le arti tecniche e a diminuire il lavoro degli uomini", Cartesio confessa che non aveva ancora preso coscienza del "vero uso" delle matematiche, limitato allora alle arti meccaniche ("mi stupivo che su fondamenti cosi fermi e solidi non si fosse ancora costruito nulla di più alto e di più importante"). ${ }^{34}$ Tuttavia la ricostruzione ex novo dell'"edificio" della filosofia su basi matematiche non fa passare in secondo piano le applicazioni pratiche, bensì dà loro nuovo incremento grazie ad una più adeguata conoscenza dei principi della fisica.

$\grave{E}$ qui che s'innesta una prospettiva etico-sociale che pare estranea alla prospettiva individualistica così enfatizzata da Cartesio e ancora ribadita all'inizio dell'ultima parte del Discours, quando il filosofo esprime piena soddisfazione per il "suo" metodo, con cui è in grado sia di risolvere questioni speculative sia di regolare la propria condotta di vita: un'impostazione tutta personale e 'privata', "poiché - egli nota con un disincanto che ci richiama Montaigne - quanto ai costumi, ognuno è cosi persuaso di essere nel giusto, che si potrebbero trovare tanti riformatori quante teste", ammesso che il compito di riformare i costumi non fosse niservato da Dio solo ai principi e ai profeti. " $\mathrm{E}$ quanto alle speculazioni, benché le mie mi piacessero molto, ho pensato che anche gli altri ne avevano, le quali forse piacevano loro anche di più". ${ }^{35}$ Ma proprio quando il discorso parrebbe definitivamente rinchiuso in quest'ottica individuale, Cartesio lo rovescia appellandosi al principio che impone di comunicare anche agli altri ciò che può essere utile all'umano consorzio:

"Ma, non appena venni in possesso di alcune nozioni generali di fisica, e, cominciando a sperimentarle in diverse difficoltà particolari, mi accorsi fin dove po-

32 Ep. 73 (L.A. SENECA, Lettere a Lucilio, intr. di L. Canali, trad. e note di G. Monti, testo latino a fronte, Milano 1989, pp. 478-479).

33 Ivi, pp. 694-701.

34 Discorso, pp. 46 e 48 (ed. Gilson, pp. 6 e 7).

35 Discorso, p. 123 (ed. Gilson, p. 61). 
tevano condurre, e quanto differissero i loro principi da quelli di cui si è fatto uso sino ad oggi, io reputai di non poterle tener nascoste senza peccare grandemente contro la legge che ci obbliga di procurare, per quanto è in noi, il bene di tutti gli uomini (le bien général de tous les hommes). Esse, infatti, mi han fatto vedere ch'è possibile pervenire a conoscenze utilissime nella vita; e che, invece di quella filosofia meramente speculativa che s'insegna nelle scuole, se ne può trovare una pratica, per la quale, conoscendo distintamente la potenza e gli effetti del fuoco, dell'acqua, dell'aria, degli astri, dei cieli e di tutti gli altri corpi a noi circostanti, [...] noi potremmo impiegarli similmente a tutti gli usi a cui sono adatti, e renderci così quasi padroni e possessori (comme maitres et possesseurs) della natura. Il che non solo è desiderabile per l'invenzione d'una infinità di congegni (artifices) che ci farebbero godere senza fatica dei frutti della terra e di tante altre comodità, ma anche, principalmente, per la conservazione della salute, la quale è, senza dubbio, il primo e fondamento di tutti gli altri beni in questa vita [...]."36

È una pagina che va meditata, perché ricca di collegamenti (si pensi allo sviluppo del'anatomia nel Seicento) e d'implicazioni. Se la critica alla filosofia delle "scuole" e l'analogia con il progetto baconiano di dominare la natura con il binomio scienza-tecnica balzano subito agli occhi, meno evidente ma non meno importante è il tema della funzione del dotto, ovvero - con termine moderno dell'intellettuale. Difatti non si tratta solo di comunicare agli altri dotti i risultati delle proprie scoperte, in linea con lo spirito della respublica litterarum, ma di far sì che tali scoperte siano utili a tutta l'umanità; e se la nozione di una legge (loi) generale cui tutti siamo assoggettati ci riporta nuovamente all'eredità stoica (ma anche al moderno giusnaturalismo), è questa funzione 'sociale' dell'uomo di scienza che rappresenta l'aspetto più nuovo, non esente - forse - da contatti con le idee dei Rosacroce. ${ }^{37}$ Senza voler intaccare il potere dei principi e della Chiesa, di origine divina, è una sorta di "terzo potere" che qui si configura, per cui il nesso primitivo tra riforma del metodo e fini morali si allarga dal perfezionamento del singolo al 'progresso' della società, dalla felicità individuale a quella che nel Settecento verrà comunemente chiamata "pubblica felicità". Se la società politica e quella ecclesiastica rimangono per Cartesio un tabù, è la "società civile" il campo in cui l'uomo di scienza - che è tale non per investitura divina ma grazie al suo "metodo" - può e anzi deve operare.

Discorso, pp. 123-125 (ed. Gilson, pp. 61-62).

37 Cfr. in proposito W.R. SHEA, Descartes and the Rosicrucians, "Annali dell'Istituto e Museo di storia della scienza di Firenze", 1979, n. 2, pp. 29-47. 